\title{
Lectin Binding Identifies a Subpopulation of Neurons in Chick Dorsal Root Ganglia
}

\author{
Sheryl A. Scott, Nimisha Patel, ${ }^{a}$ and Joel M. Levine \\ Department of Neurobiology and Behavior, State University of New York at Stony Brook, Stony Brook, New York 11794
}

\begin{abstract}
We screened a variety of lectins with different sugar specificates to determine whether subpopulations of dorsal root ganglion (DRG) neurons in the chick can be distinguished by the carbohydrates they express. Of the 15 lectins tested only those that recognize $\mathrm{N}$-acetylgalactosamine (galNac) residues labeled a subset of DRG neurons. For example, Dolichos biflorus (DBA) labeled a population of small-diameter neurons in the dorsomedial DRG and their terminals in the dorsal horn in hatchling chicks. Staining of live neurons in vitro demonstrated that DBA was binding to the cell surface. Labeling first appeared in sensory neurons at about St.38 (E12) and in dorsal horn laminae 1 and 2 at about St.42 (E16). Fainter labeling appeared somewhat later in lamina 3, after hatching. Labeling of the tissue sections was eliminated by chloroform : methanol extraction and reduced by $\alpha$ - $N$-acetylgalactosaminidase digestion, but survived trypsinization. Together these results suggest that a subset of DRG neurons in the chick can be identified by the presence of a cell surface glycoconjugate, perhaps a glycolipid, containing terminal $\alpha$-linked galNac residues.
\end{abstract}

Dorsal root ganglia (DRGs) contain a diversity of sensory neurons that can be divided into functional subsets on the basis of size (Lawson, 1979), ultrastructural and cytochemical characteristics (Rambourg et al., 1983; Sommer et al., 1985), physiological properties (Harper and Lawson, 1985; Rose et al., 1986), peptide content (Hökfelt et al., 1976; Leah et al., 1985; Price, 1985; New and Mudge, 1986), and central and peripheral connections (Brown, 1981). In addition, subpopulations of DRG neurons can be recognized by the constellation of carbohydrates that they express (Dodd et al., 1984; Dodd and Jessell, 1985, 1986; Fischer and Csillik, 1985; Jessell and Dodd, 1985; Lawson et al., 1985; Streit et al., 1985, 1986; Marusich et al., 1986; Mori, 1986; Nakagawa et al., 1986; Plenderleith et al., 1988). While much is known about the overall development of peripheral (reviewed in Scott, 1987) and central (Smith, 1983; Altman and Bayer, 1984; Lee et al., 1988; Smith and Frank, 1988; Davis et al., 1989) connections of DRGs, little is known about the specificity with which identified subpopulations of sensory neurons grow to and synapse with their peripheral and central targets. Glycoconjugates that identify various subsets of

\footnotetext{
Received Dec. 16, 1988; reviscd May 8, 1989; accepted July 11, 1989.

We thank Deborah Lawson for expert technical assistance. This work was supported by NIH grants NS16067 (S.A.S.) and NS21198 (J.M.L.) and a Fogarty Senior International Fellowship (S.A.S.)

Correspondence should be addressed to Sheryl A. Scott at the above address

a Present address: New York University School of Medicine, New York, NY 10016.

Copyright (C) 1990 Society for Neuroscience $0270-6474 / 90 / 010336-10 \$ 02.00 / 0$
}

sensory neurons might serve as useful markers for investigating these problems.

Cell surface glycoconjugates are likely to serve important functions in neural development, such as cell-cell recognition and adhesion (Edelman, 1984; Fischer and Csillik, 1985; Streit et al., 1985; Dodd and Jessell, 1986; Peinado et al., 1987), axon guidance (Dodd and Jessell, 1986), and synapse formation and/ or maintenance (Fischer and Csillik, 1985; Grunwald et al., 1985). Few of these functions have becn directly demonstrated in vivo (Fraser et al., 1988; Landmesser et al., 1988). It would be of interest to examine the role of various glycoconjugates, such as those that define subpopulations of sensory neurons, by perturbing their function during critical embryonic stages. Such manipulations should be possible in the chick, where embryos are readily accessible to experimental intervention (cf. Landmesser el al., 1988).

The glycoconjugates of chick sensory neurons, however, have not been studied in detail (Scott et al., 1987). We have, therefore, screened a battery of lectins to determine whether any carbohydrate is expressed selectively by a unique subset of DRG neurons in the chick, and if so, to determine the type of glycoconjugate involved and the embryonic stage at which it first appears. We show here that a subpopulation of small-diameter sensory neurons in the chick and their terminals in the dorsal horn can be identified by the presence of a cell surface glycoconjugate, perhaps a glycolipid, containing terminal $\alpha$-linked $N$-acetylgalactosamine (galNac) residues. This glycoconjugate first appears in sensory neurons at about St.38 (E12) and in dorsal horn laminae 1 and 2 at about St.42 (E16). Fainter labeling appears somewhat later in lamina 3, after hatching. Some of these results have been presented previously in abstract form (Patel and Scott, 1988).

\section{Materials and Methods}

Animals. White Leghorn chick eggs were incubated at $38^{\circ} \mathrm{C}$ in a forceddraft incubator until the desired stage. Embryos were staged according to Hamburger and Hamilton (1951). Chicks were hatched from eggs in the laboratory and were housed in brooders at $36^{\circ} \mathrm{C}$ until sacrifice.

Cultures. Cultures were established from E14 lumbosacral (LS) DRGs. DRGs were dissociated in $0.1 \%$ trypsin (Worthington, Freehold, NJ) and grown for $48 \mathrm{hr}$ on polyornithine-laminin coated dishes in Ham's F14 medium (Imperial Laboratories, Salisbury, UK) supplemented with $10 \%$ horse serum and $10 \mu \mathrm{g} / \mathrm{ml} \mathrm{NGF}$.

Lectins. Lectins used in this study are listed in Table 1. Horseradish (HRP)-conjugated Arachis hypogaea (PNA) and Dolichos biflorus (DBA) and fluorescein (FITC)-conjugated Bandeiraea simplicifolia- $\mathrm{A}_{4}$ (BSI$\mathrm{A}_{4}$ ), Concanavalin A (Con A), DBA, Glycine max (SBA), Phaseolus limensis (LBL), Ulex europaeus (UEA), Vicia villosa- $\mathrm{B}_{4}$ (VVA- $\left.\mathrm{B}_{4}\right)$, and rhodamine (Rh)-conjugated Triticum vulgaris (WGA) were purchased from Sigma (St. Louis, MO). Biotinylated Lens culinaris (LCA), Phaseolus vulgaris erythroagglutinin (PHA-E), Phaseolus vulgaris leukuagglutinin (PHA-L), Pisum sativum (PSA), and Ricinus communis-I (RCA- 
I) were from Vector (San Francisco, CA). Biotinylated Sophora japonica (SJA) was purchased from both Sigma and Vector. Biotinylated LBL was obtained from E-Y Labs (San Mateo, CA).

Histology. Hatchling chicks were anesthetized with an overdose of xylazine and ketamine. Chicks and embryos were perfused with either $6 \%$ dextran or $0.1 \mathrm{~m}$ phosphate buffered saline (PBS), $\mathrm{pH} 7.2$, followed by $4 \%$ paraformaldehyde in PBS, and postfixed for $2 \mathrm{hr}$. The LS spinal cord and DRGs were dissected, rinsed in PBS, and cryoprotected in $20 \%$ sucrose.

Cryostat sections $12 \mu \mathrm{m}$ thick were air-dried onto slides and dipped in $0.4 \%$ paraformaldehyde in PBS for $15 \mathrm{sec}$. For lectin binding studies slides were rinsed in PBS containing $0.1 \mathrm{mM} \mathrm{CaCl}, \mathrm{MgCl}_{2}$, and $\mathrm{MnCl}_{2}$ and incubated overnight at $4^{\circ} \mathrm{C}$ with a lectin diluted to $20 \mu \mathrm{g} / \mathrm{ml}$ with the same buffer. HRP-conjugated lectins were visualized with $0.05 \%$ diaminobenzidine (DAB) (Wako Chemicals, Dallas, TX) containing $0.002 \% \mathrm{H}_{2} \mathrm{O}_{2}$ in PBS. Biotinylated lectins were visualized with either Texas Red conjugated avidin or avidin-biotin complex from Vector. Fluorescently labeled sections were mounted in glycerol containing 0.4 $\mathrm{mg} / \mathrm{ml} p$-phenylenediamine. As controls, slides were incubated overnight in PBS without lectin or in lectin containing $0.1 \mathrm{M}$ fucose, mannose, galactose, glucose, or $N$-acetylgalactosamine.

Living sensory neurons in vitro were incubated with FITC-conjugated DBA (20-50 mg/ml) in L-15 medium (Gibco, Paisley, Scotland) for 1 $\mathrm{hr}$, washed, and fixed for $20 \mathrm{~min}$ in $4 \%$ paraformaldehyde in $0.12 \mathrm{M}$ phosphate buffer

To visualize, substance $P$ (SP)-like immunoreactivity slides were incubated overnight at $4^{\circ} \mathrm{C}$ in anti-SP (Accurate Chemical Corp., Westbury, NY) diluted 1:500 and reacted with the PAP procedure (Sternberger, 1986)

Characterization of the glycoconjugate. To extract lipids from sections, slides were incubated in a mixture of chloroform : methanol (2: 1) for $20 \mathrm{~min}$ at $-20^{\circ} \mathrm{C}$ and air-dried for $15 \mathrm{~min}$ before incubation with DBA or anti-SP. Other slides were incubated in $0.05 \%$ trypsin in PBS at $37^{\circ} \mathrm{C}$ for $10 \mathrm{~min}$ and rinsed with cold PBS before incubation with DBA or anti-SP. For glycosidase digestion, cryostat sections of unfixed tissue were incubated with $\beta$ - $N$-acetylhexosaminidase from jack bean or $\alpha$-N-acetylgalactosaminidase from chicken liver (Sigma) (36 milliunits $/ 40 \mu$ l citrate buffer, $\mathrm{pH} 4.0$ ) for $5 \mathrm{hr}$ at $37^{\circ} \mathrm{C}$.

Cell size measurement. The size of DBA-labeled neurons in DRG LS2 of 1 -week-old chicks was determined by 2 methods. In 1 chick ( $L 41$, Fig. 3) every fourth section was photographed and enlarged at a standard magnification. Each neuronal profile was scored as unlabeled, or as light, medium, or darkly stained with DBA. The effective diameter of each profile was calculated from the area of the profile on the photograph measured with a digitizing tablet. The number of labeled neurons and the histogram of their sizes were then determined using the regressive translation method of Rose and Rohrlich (1987). In 2 additional chicks ( $L 4 U$ and $L 48$, Fig. 3 ) every neuron profile containing a nucleus was scored for DBA staining as above in several widely spaced $(>50 \mu \mathrm{m})$ sections. Sections were viewed with a $100 \times$ oil immersion objective, and 2 diameters (perpendicular to each other) were measured with an eyepiece graticule; the mean of the 2 diameters was taken as the average diameter.

Targets of labeled neurons. To determine the peripheral targets of lectin-labeled neurons, hatchlings were anesthetized with ketamine $(0.03$ $\mathrm{ml})$ and xylazine $(0.06 \mathrm{ml})$. The cutaneous femoralis lateralis nerve or sartorius nerve was injected with $25 \% \mathrm{Rh}$-dextran amine (Molecular Probes, Eugene, OR) and crushed distal to the injection. Three to four days later animals were anesthetized, perfused, and dissected as described above.

\section{Results}

\section{Patterns of lectin binding}

The lectins studied, their apparent sugar specificities, and patterns of binding in DRGs and spinal cord of 1-2-day chick hatchlings are listed in Table 1 and illustrated in Figure 1. Most of the 15 lectins tested did not specifically label subsets of sensory neurons in DRGs. Some lectins (Group A, Table 1), such as UEA, which binds to fucose, labeled white matter in the spinal cord, but did not label any DRG neurons (Fig. 1, $A, E$ ). Others (Group B, Table 1), such as RCA-I and LCA, which bind to galactose and mannose, respectively, formed a mesh-
Table 1. Lectins used in this study

\begin{tabular}{lll} 
Lectin & $\begin{array}{l}\text { Nominal sugar } \\
\text { specificity }^{a}\end{array}$ & $\begin{array}{l}\text { Staining } \\
\text { pattern }^{b}\end{array}$ \\
\hline Phaseolus limensis (LBL) & galNac & $\mathrm{A}$ \\
Sophora japonica (SJA) & galNac, gal & $\mathrm{A}$ \\
Ulex europaeus (UEA) & fuc & $\mathrm{A}$ \\
Lens culinaris (LCA) & man & $\mathrm{B}$
\end{tabular}

Phaseolus vulgaris

erythroagglutinin (PHA-E)

oligosaccharide B

Phaseolus vulgaris

leukoagglutinin (PHA-L)

Pisum sativum (PSA)

Ricinus communis-I (RCA-I)

Arachis hypogaea (PNA)

Concanavalin A (Con A)

Triticum vulgaris (WGA)

Bandeiraea simplicifolia- $\mathrm{A}_{4}$ (BSI-A $)_{4}$

Dolichos biflorus (DBA)

Glycine max (SBA)

Vicia villosa- $\mathrm{B}_{4}\left(\mathrm{VVA}-\mathrm{B}_{4}\right)$

$\begin{array}{ll}\text { oligosaccharide } & \text { B } \\ \text { man } & \text { B } \\ \text { gal, galNac } & \text { B } \\ \text { gal } \beta(1-3) \text { galNac } & \text { C } \\ \text { man, glc } & \text { C } \\ \text { glcNac, neuNac } & \text { C }\end{array}$

"fuc, fucose; gal, galactose; galNac, $N$-acetylgalactosamine; glc, glucose; glcNac, $N$-acetylglucosamine; man, mannose; neuNac, neuraminic (sialic) acid.

${ }^{b}$ Examples of the staining patterns are shown in Figure 1. Group $A$ corresponds to Figure $1, A, E$; Group $\mathrm{B}$ to Figure $1, B, F$; Group $\mathrm{C}$ to Figure $1, C, G$; and Group D to Figure $1, D, H$.

work of labeling throughout the gray matter of the spinal cord and brilliantly outlined all DRG neurons (Fig. $1, B, F$ ), presumably labeling the satellite cell sheath or basal lamina that surrounds each neuron (Lieberman, 1976). Still other lectins (Group C, Table 1), such as Con A, which binds to mannose and glucose, and WGA, which binds to $\mathrm{N}$-acetylglucosamine and neuramic acid (Fig. 1, $C, G$ ), labeled all DRG neurons uniformly. In each case the generalized staining was blocked by the appropriate sugar. None of these lectins discriminated between sympathetic and DRG neurons. With each of these lectins the labeling in sympathetic ganglia was identical to that in the DRGs.

In contrast, most of the lectins that recognize galNac residues, such as DBA, labeled a subpopulation of neurons in LS DRGs and a discrete projection in the dorsal horn (Fig. 1, $D, H$ ). All of these lectins (Group D, Table 1) failed to label sympathetic neurons, and thus each appeared to identify a subset of sensory neurons specifically. The galNac-binding lectins were studied further to learn more about the population of DRG neurons that express terminal galNac residues, the type of glycoconjugate involved, and the time at which the carbohydrate appears during embryonic development. Other lectins that did not selectively label sensory neurons (Groups A-C, Table 1) were not studied further.

\section{Characterization of the lectin-binding moiety}

The staining pattern varied among the 6 galNac-binding lectins that we tested (Table 1). DBA and BSI- $\mathrm{A}_{4}$ gave the most specific and intense labeling, producing nearly identical staining patterns. Both labeled a subset of small-diameter neurons in the dorsomedial DRG and dorsal horn laminae 1 and 2, as well as Lissauer's tract (Martin, 1979). The same overall pattern of labeling was seen throughout all LS levels; other spinal levels were not examined. Staining by VVA-B ${ }_{4}$ and SBA was similar to DBA and BSI- $A_{4}$, except that these lectins also nonspecifically 

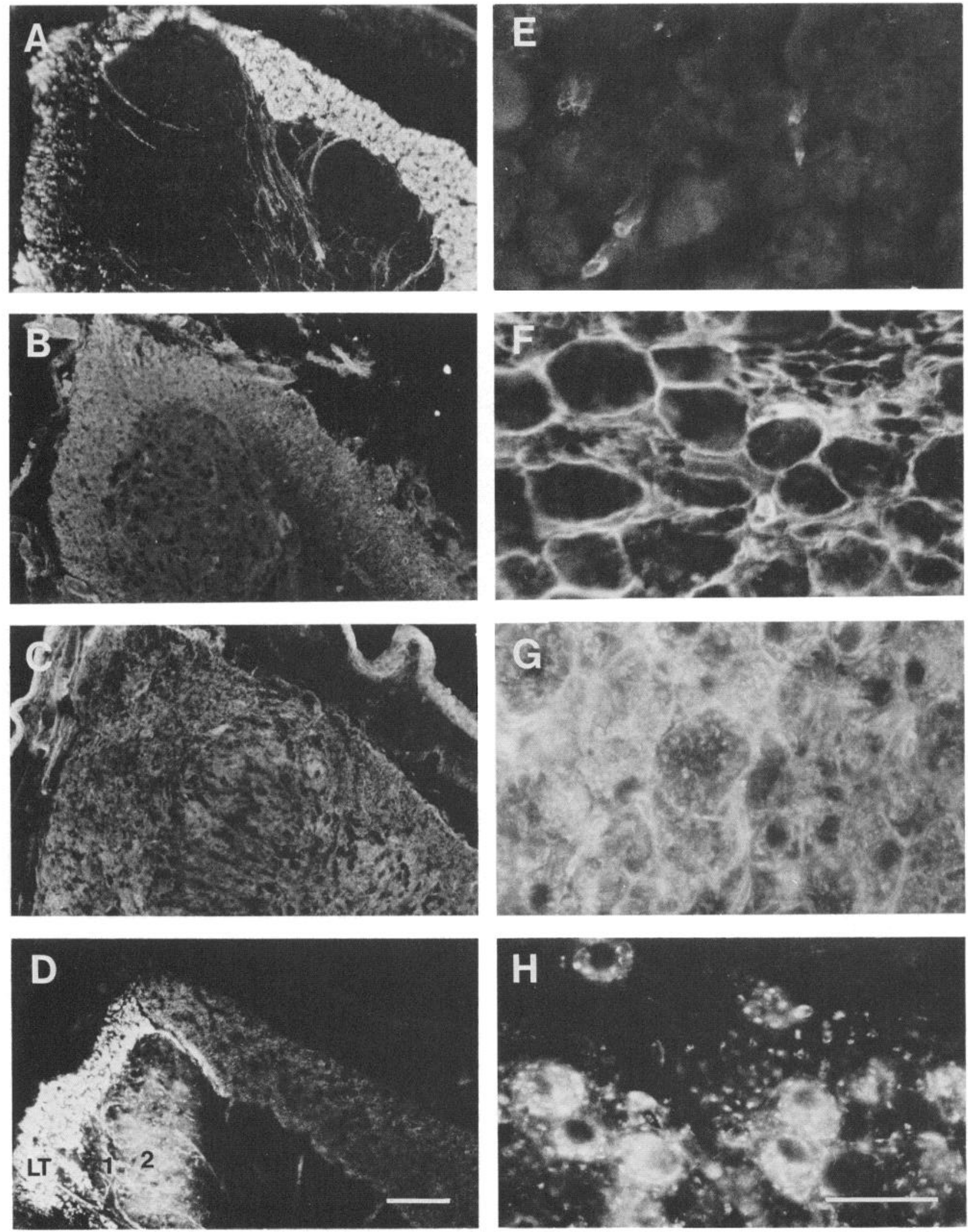

Figure 1. Lectin binding in dorsal horn (left) and dorsal root ganglia (right) of 1-2-d chick hatchlings. $A, E$, UEA; $B, F$, RCA; $C, G$, WGA; $D$ $H$, DBA. Only DBA and other lectins that recognize galNac residues label a subset of neurons and their processes in Lissauer's tract (LT) and dorsal horn laminae 1 and 2. Scale bar: $100 \mu \mathrm{m}$ for the dorsal horn; $50 \mu \mathrm{m}$ for the neurons. 

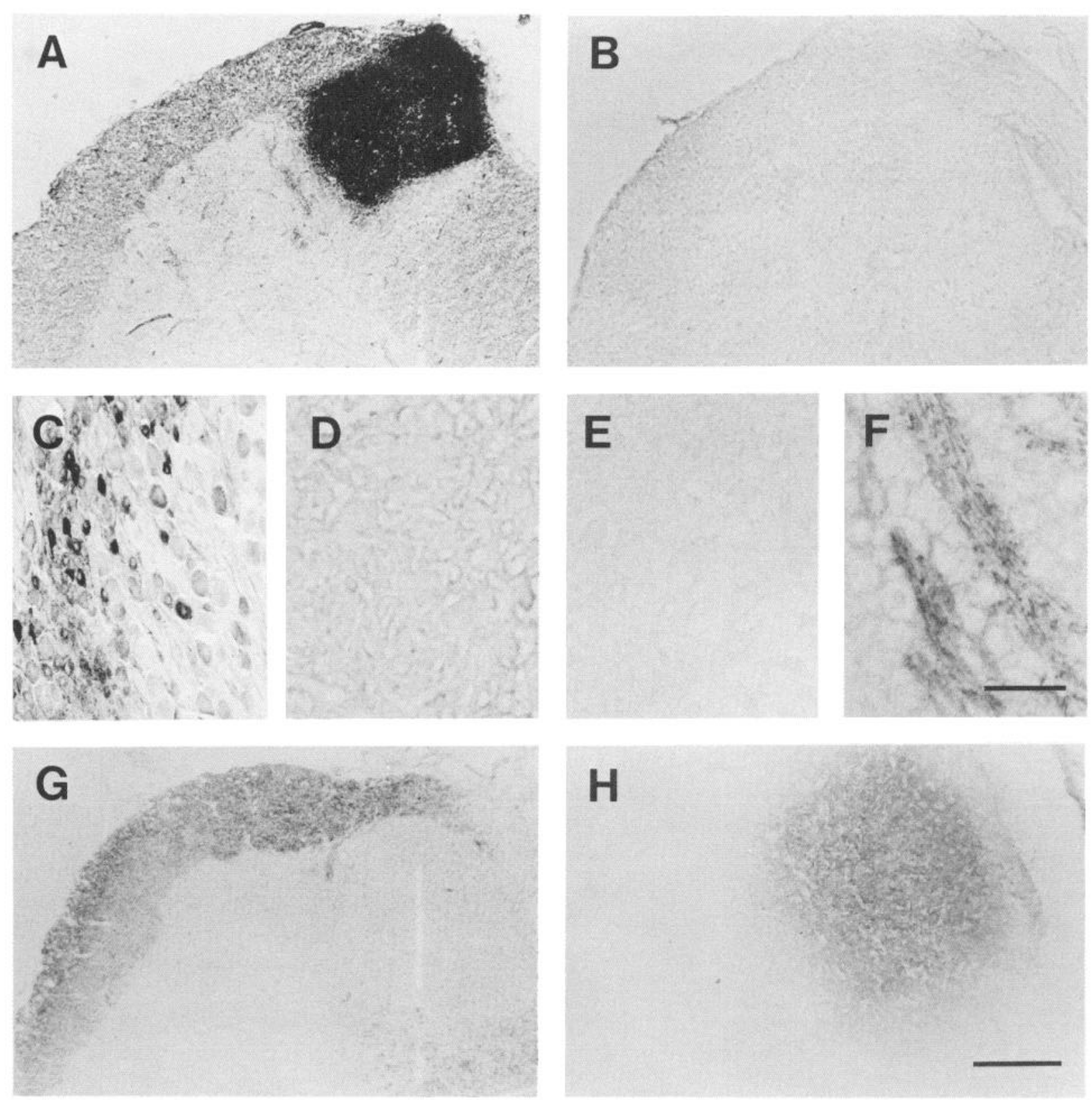

Figure 2. Staining by DBA in dorsal horn $(A, B, G, H)$ and lumbosacral dorsal root ganglia $(C-F)$ of $1-2-$ d chick hatchlings. $A$, $C$, Untreated control sections stained with DBA. $B, D$, DBA staining is blocked with excess galNac. $E, G$, DBA staining is eliminated by chloroform: methanol extraction. $F, H$, DBA staining is reduced by digestion with $\alpha-N$-acetylgalactosaminidase. Scale bar: $200 \mu \mathrm{m}$ for the dorsal horns; $100 \mu \mathrm{m}$ for the neurons.

labeled some white matter in the cord. All of these lectins appeared to label a similar subpopulation of DRG neurons. However, further experiments are required to determine whether all of these lectins label the identical subset of neurons. Surprisingly, 2 lectins that are reported to be specific for galNac, LBL (Galbraith and Goldstein, 1970) and SJA (Poretz et al., 1974), failed to label DRG neurons. Possible reasons for this are discussed below.

DBA is known to recognize terminal galNac residues (Hammarstrom et al., 1977). In chick spinal cord and DRGs, DBA binding was completely blocked by excess galNac (Fig. 2, B, D) but not by other sugars (fucose, mannose, galactose, and glucose). Thus, as expected, DBA appeared to bind specifically to terminal galNac residues in chick neural tissue.
The galNac residues recognized by DBA appeared to be associated with a glycolipid rather than a glycoprotein. DBA staining was eliminated completely by chloroform: methanol extraction (Fig. 2, $E, G$ ). The residual staining seen in the white matter following lipid extraction (Fig. $2 G$ ) was nonspecific and was not blocked by excess galNac. Chloroform : methanol extraction had no detectable effect on immunoreactivity of the peptide SP. Trypsin digestion, on the other hand, eliminated SP staining, but left DBA binding intact (data not shown). Thus, the galNac residues labeled by DBA appeared to be linked to a glycolipid, although further experiments are required to prove this definitively.

Terminal galNac residues can be linked in carbohydrates in 3 different ways: (1) as an $\alpha$-linked terminal sugar of a oligo- 
Figure 3. Histograms of the size of DBA-labeled neurons in DRG LS2 of 3 1-week-old chicks determined by the method of Rose and Rohrlich (1987) $(L 41)$ and by measuring the diameters directly ( $L 40$ and $L 48$ ). The larger, outer histograms show the size spectrum of all neurons in the DRGs. The smaller, shaded histograms show the size of light, medium, and darkly DBA-stained neurons in each chick. Arrowheads show the overall mean diameter and the mean of each staining category. Note that medium and darkly stained cells tend to be small.

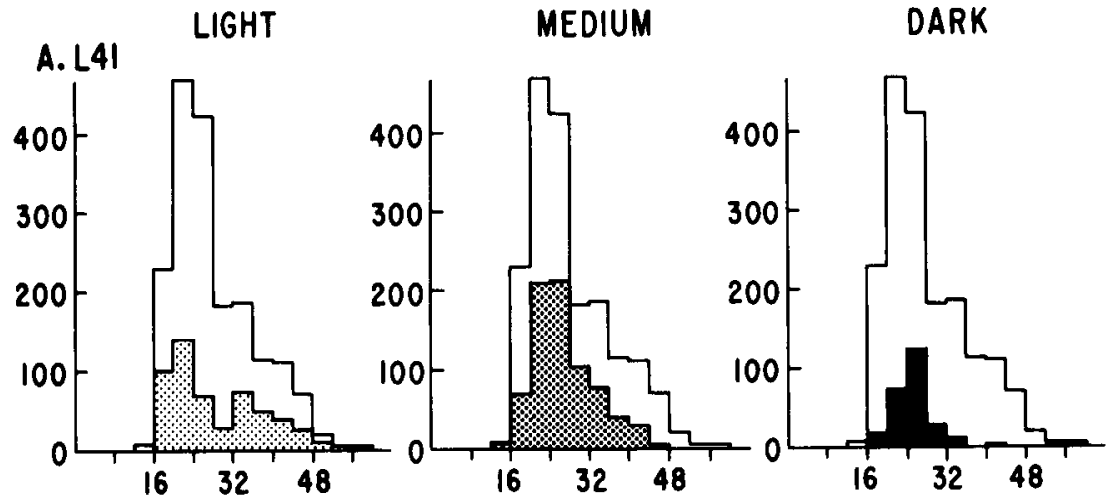

B. L40
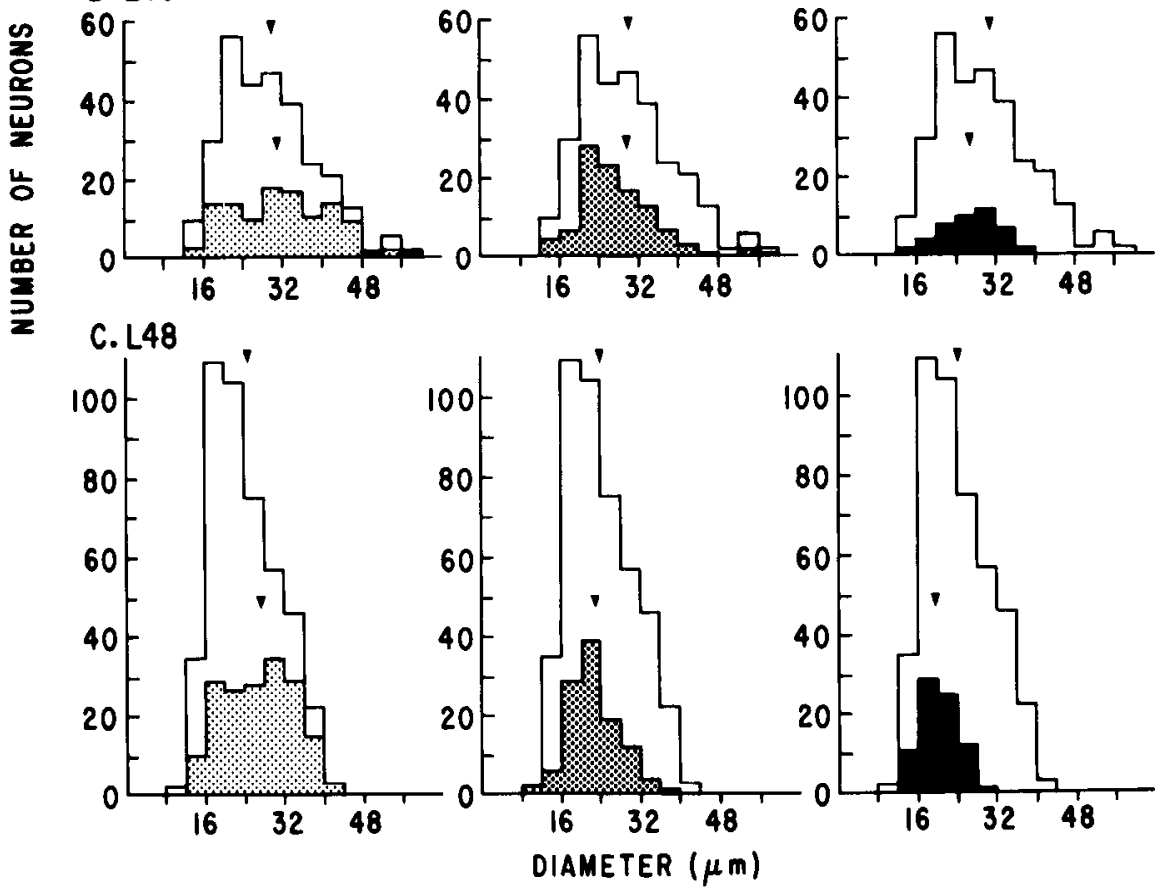

saccharide, (2) as a $\beta$-linked terminal sugar, or (3) $\mathrm{O}$-linked to a threonine or serine residue of a protein. DBA can recognize galNac in all 3 conformations. It is unlikely, however, that the galNac residues recognized by DBA are O-linked since they appear to be associated with a glycolipid and not with a glycoprotein. To distinguish between $\alpha$ - and $\beta$-linkages, sections were digested with specific glycosidases prior to staining. DBA binding was eliminated from DRG somata and substantially reduced in the dorsal horn and intraganglionic axons by $\alpha-N$ acetylgalactosaminidase digestion (Fig. 2, $F, H$ ). In contrast, staining was unaffected by $\beta$ - $N$-acetylhexosaminidase, suggesting that the galNac residues specifically recognized by DBA on sensory neurons are in the $\alpha$-linked conformation and are indeed in the terminal position. This most likely accounts for the failure of SJA, which prefers terminal $\beta$-galNac residues (Poretz et al., 1974), to label DRG neurons. Some staining was still apparent in dorsal horn and intraganglionic axons following $\alpha$ - $N$-acetylgalactosaminidase digestion, however. Possible reasons for the residual staining are discussed below.

\section{Characterization of lectin-positive neurons}

Superficial examination of lectin-stained sections suggested that DBA intensely labeled only small-diameter neurons, and more lightly labeled neurons of all size classes (see inset, Fig. 6). To quantify this, labeled and unlabeled neurons were measured in DRG LS2 in week-old chicks $(n=3)$, as described in Materials and Methods. This analysis confirmed our initial impression, as shown in Figure 3. DBA intensely labeled approximately $16 \%$ of DRG neurons (range $=14-17 \%$ ). These neurons were clearly small diameter (mean $=22.8 \mu \mathrm{m} \pm 5.5, \mathrm{SD})$; no large, darkly labeled neurons were observed. An additional $70 \%$ of neurons (range $=64-75 \%$ ) showed light or medium staining. Lightly labeled neurons spanned the entire size spectrum (mean $=29.2$ $\mu \mathrm{m} \pm 8.9, \mathrm{SD}$ ), while medium stained neurons were mostly small diameter $($ mean $=25.5 \mu \mathrm{m} \pm 7.3, \mathrm{SD})$.

To identify the targets of the lectin-labeled neurons, cutaneous and muscle afferents were retrogradely labeled with rhodaminedextran amine prior to staining with FITC-conjugated DBA, VVA- ${ }_{4}$, or BSI-A $A_{4}$. Double-labeled DRG neurons were found in DRGs LS1 and LS2 after injection of tracer into either the cutaneous femoralis lateralis (Fig. 4, $A, B$ ) or sartorius nerve (Fig. $4, C, D$ ), showing that lectin-binding neurons project to targets in both skin and muscle.

The staining observed in sections of DRGs appeared to be intracellular. In order to determine whether the galNac-containing carbohydratc was also expressed on the cell surface, 

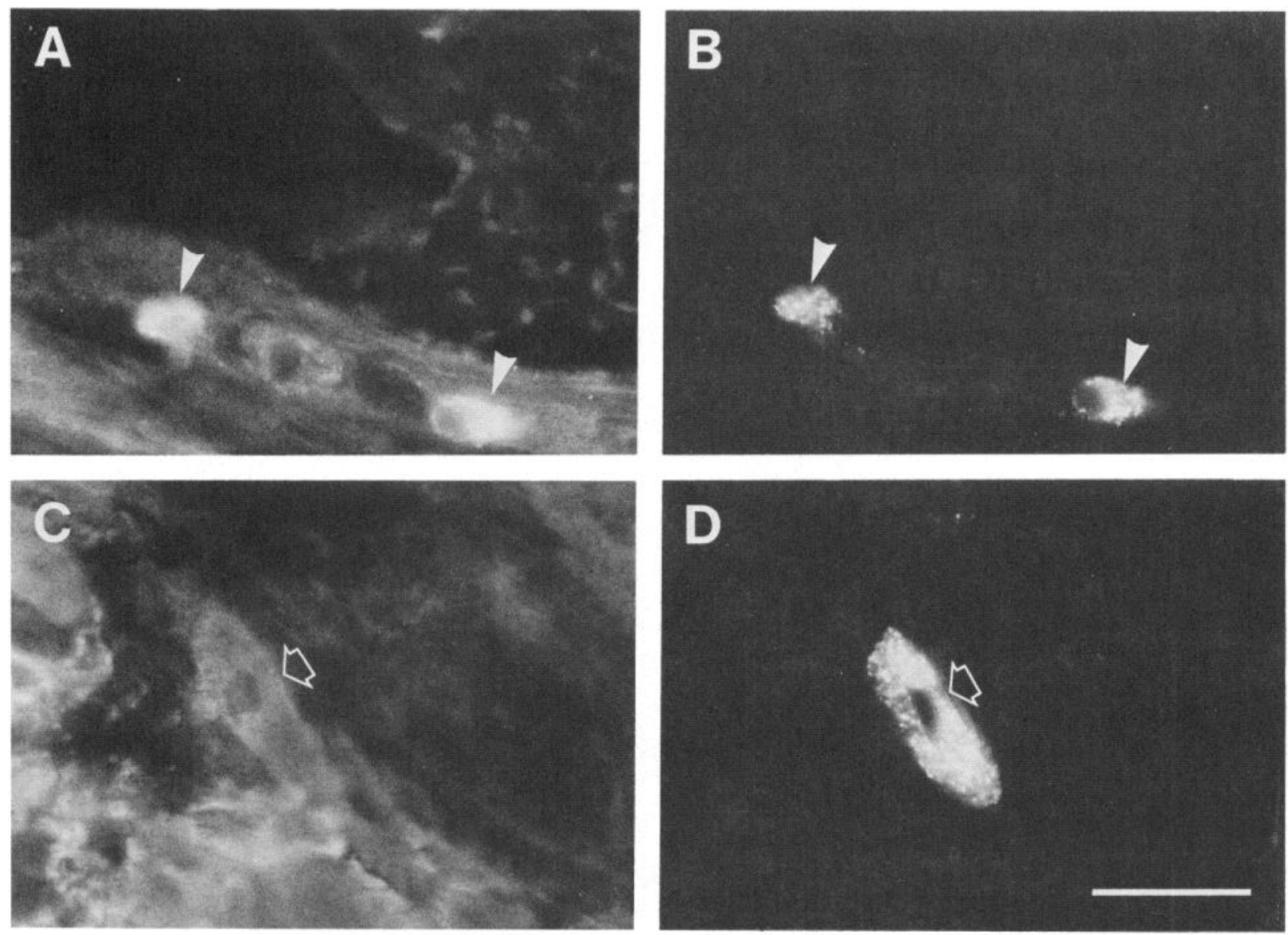

Figure 4. Determination of targets of DBA-labeled neurons. $A$ and $C$ (arrows) show DRG neurons stained with FITC-conjugated DBA. $B$ and $D$ (arrows) show the same neurons retrogradely labeled with rhodamine-dextran amine injected into the cutaneous femoralis lateralis nerve $(B)$ or sartorius muscle nerve $(D)$. Thus, DBA labels DRG neurons with targets in both skin and muscle. Scale bar, $50 \mu \mathrm{m}$.

living DRG neurons were stained in vitro with FITC-conjugated DBA. The somata and neurites of DRG neurons were distinctly labeled with DBA, as illustrated in Figure 5, while Schwann cells and other non-neuronal cells were not. On average, ap- proximately $75 \%$ of neurons were labeled, about the same proportion as in sections of DRGs (Fig. 3).

Together these results show that a subpopulation of LS DRG neurons in the chick and their terminals in the dorsal horn can
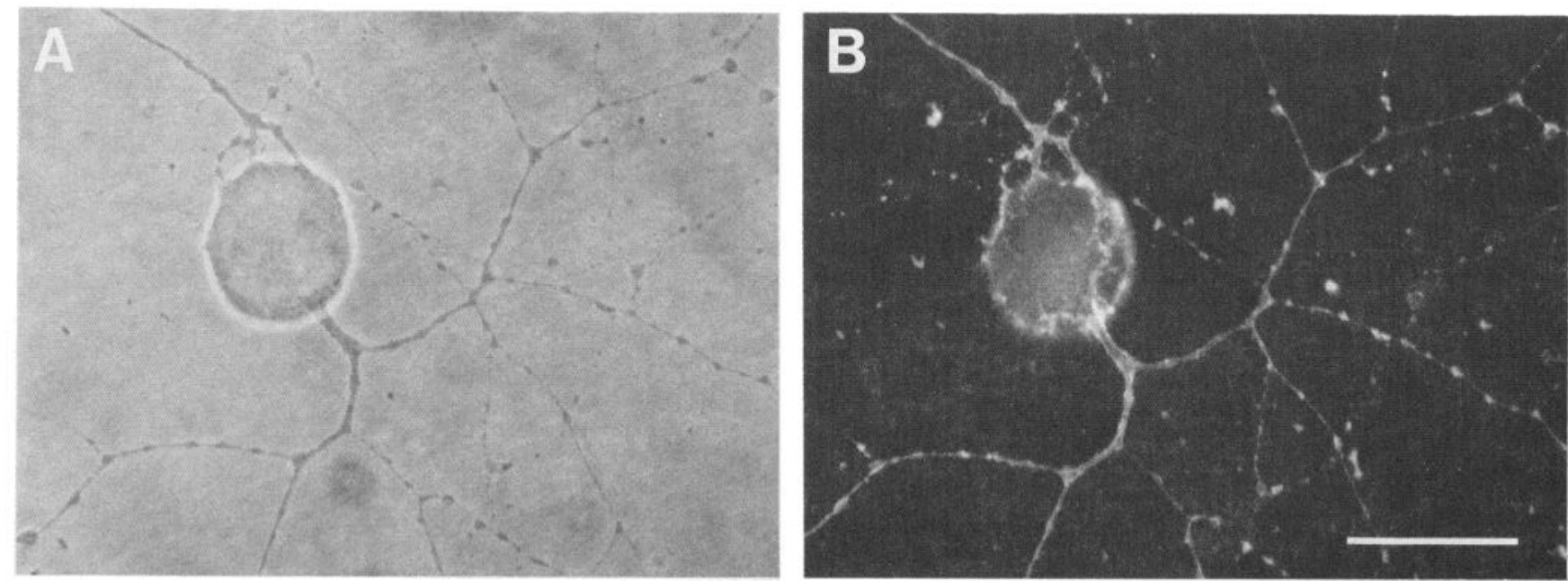

Figure 5. Cell surface staining by DBA. $A$, Phase contrast micrograph of a DRG neuron grown in dissociated cell culture. $B$, The same neuron is stained with FITC-conjugated DBA. Scale bar, $40 \mu \mathrm{m}$. 
Figure 6. Development of lectin binding in lumbosacral DRGs and spinal cord. $A$, Spinal cord and DRG from a St.38 (E12) embryo stained with DBA. A few DRG neurons are labeled at this stage (inset), while terminals in the dorsal horn are not. $B, C$, Spinal cord $(B)$ and DRG $(C)$ of a St.43 embryo stained with DBA. Terminals in the lateral dorsal horn are clearly labeled, as are many small neurons in the dorsomedial DRG. Scale bar: $200 \mu \mathrm{m}$ in $A$ and $B ; 100 \mu \mathrm{m}$ in $C$; and $20 \mu \mathrm{m}$ in the inset.
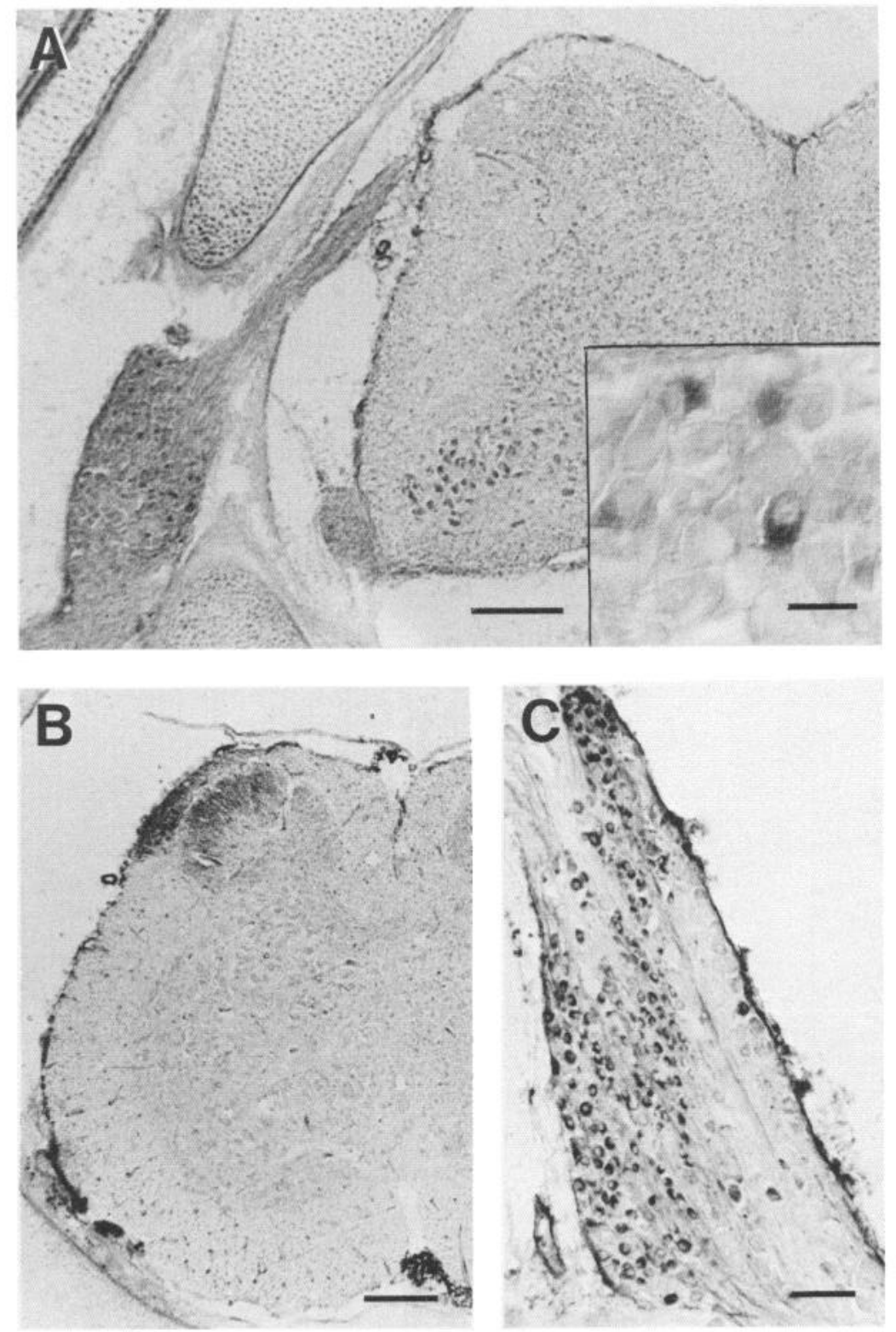

be identified by the presence of a cell surface glycoconjugate containing terminal $\alpha$-linked galNac residues, and that the galNac residues are likely to be associated with a glycolipid.

\section{Development of lectin binding}

The development of DBA binding was examined in embryos beginning at St.35 (E9). At this stage no specific labeling could be detected in either DRG or spinal cord. By St.38 (E12) a few distinctly labeled neurons were present in DRGs in 1 of the 2 embryos examined, but terminals in the dorsal horn were not obviously labeled (Fig. $6 \mathrm{~A}$ ). Labeled terminals in the dorsal horn first became apparent at about St.42 (E16), when labeling in the DRGs resembled the mature pattern. An example of DBA staining in a St. 43 embryo is shown in Figure $6 \mathrm{~B}$. At all stages studied, specific labeling was blocked by excess galNac and eliminated by chloroform : methanol extraction, suggesting that from their earliest appearance the terminal galNac residues in sensory neurons are associated with a glycolipid.
During the next week DBA staining increased in dorsal horn laminae 1 and 2 . In addition, by 1 week after hatching, faint staining was occasionally detected ( $2 / 4$ chicks) in lamina 3 , as shown in Figure 7.

\section{Discussion}

We have shown that a subpopulation of small-diameter neurons in LS DRGs and their terminals in laminae 1 and 2 (and to a lesser extent lamina 3) can be distinguished by the presence of a cell surface glycoconjugate containing terminal $\alpha$-linked galNac residues; the galNac residues appear to be linked to a glycolipid. This glycoconjugate first becomes detectable in DRG neurons at about St.38 (E12) and subsequently appears in sensory terminals of the dorsal horn and in Lissauer's tract.

Although we tested lectins specific for a number of other monosaccharides, we found that only galNac-binding lectins identified a carbohydrate that was selectively expressed by a subpopulation of DRG neurons in hatchling chicks. We cannot 


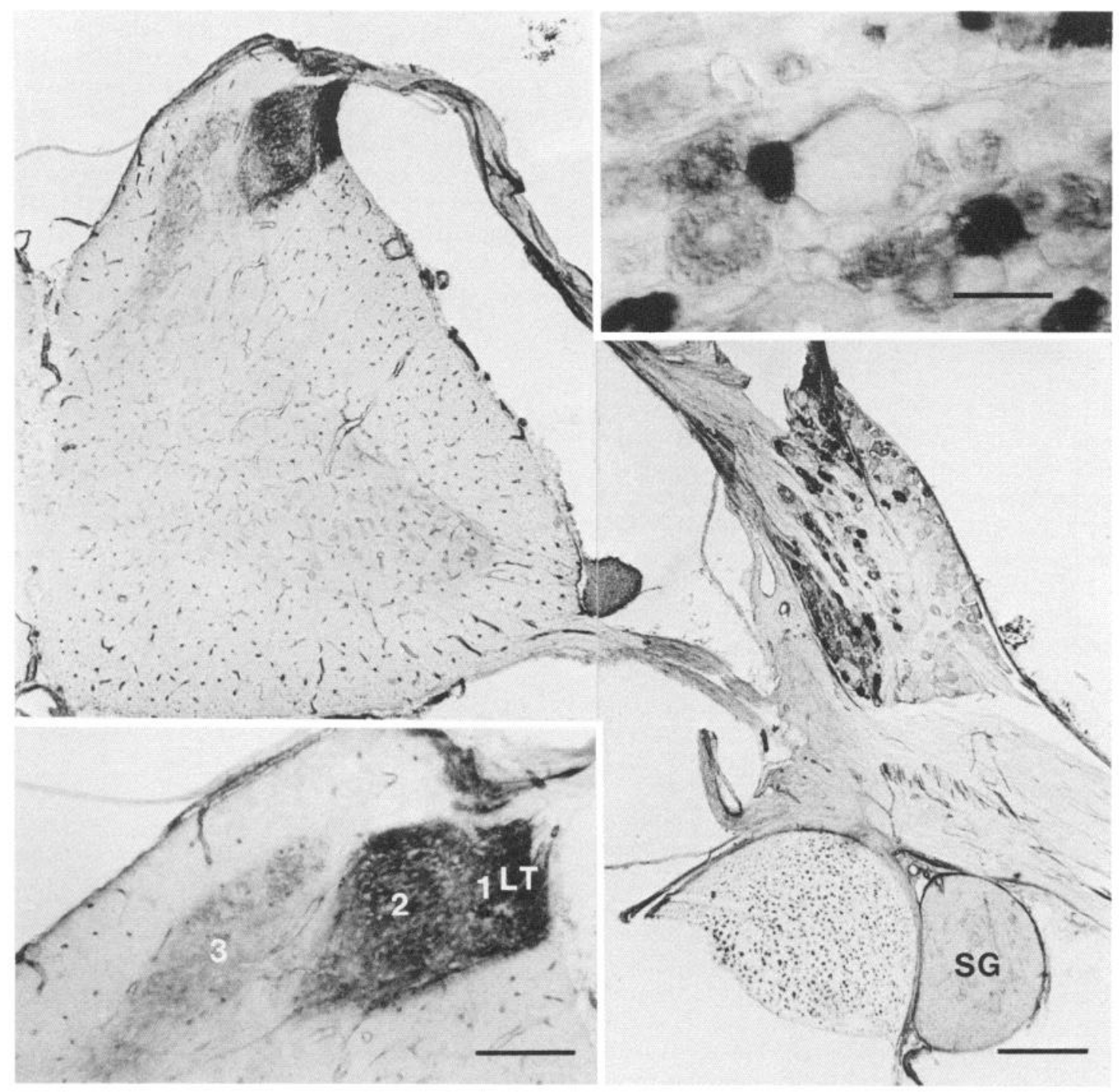

Figure 7. Spinal cord and dorsal root ganglion of a 1-week-old chick stained with DBA. Insets show DRG neurons and dorsal horn at higher magnification. By 1 week after hatching, light labeling appears in dorsal horn lamina 3, in addition to the darker staining in laminae 1 and 2 . Note that the large ventrolateral DRG neurons and neurons in the sympathetic ganglion $(S G)$ are not stained. $L T$, Lissauer's tract. Scale bar: $300 \mu$ m; $50 \mu \mathrm{m}$ for inset of DRG neurons, and $200 \mu \mathrm{m}$ for inset of dorsal horn.

rule out the possibility, however, that other glycoconjugates might be transiently expressed by subpopulations of sensory neurons at earlier stages, as occurs elsewhere in the developing nervous system (Levine et al., 1984; Grunwald et al., 1985; Yamamoto et al., 1985; Dodd and Jessell, 1986; Schwarting et al., 1987). The possibility also exists that other populations of DRG neurons express carbohydrates with unique subterminal structures, which we would not have detected in the present lectin-binding studies (cf. Scott et al., 1987).

DBA labeling was eliminated from DRG somata and reduced substantially in the dorsal horn by digestion with $\alpha$-N-acetylgalactosaminidase. The residual staining in the dorsal horn can be accounted for by at least 3 possibilities. First, it is possible that all of the staining would be eliminated with longer or stronger digestion. Second, perhaps some of the galNac is, in fact, $\beta$-linked, but any reduction in DBA staining resulting from $\beta$-hexosaminidase digestion was masked by the intense staining of the remaining $\alpha$-linked galNac residues. This seems unlikely, however, since SJA, which prefers $\beta$-linked galNac residues (Po- retz et al., 1974), did not label the dorsal horn. Third, it is possible that digestion of the terminal galNac residues exposed additional galNac residues in the penultimate position, which then stained with DBA. At present we cannot distinguish among these possibilities. Nevertheless, it appears that the majority of galNac residues recognized by DBA are in the $\alpha$-linked conformation.

Carbohydrates containing terminal galNac residues are found in preimplantation murine embryos (Shevinsky et al., 1982; Kannagi et al., 1983), embryonic rodent brain (Yamamoto et al., 1985), subpopulations of intermediate- and large-diameter sensory neurons in mammals (Dodd et al., 1984; Jessell and Dodd, 1985), and at the neuromuscular junction in a large number of species (Scott et al., 1988). In each of these cases, however, the terminal galNac is $\beta$-linked, rather than $\alpha$-linked, as we found in the chick.

There were some differences in the labeling of DRG neurons and their central terminals among the 6 galNac-specific lectins that we tested. VVA-B $B_{4}$ and SBA showed more nonspecific la- 
beling, and SJA and LBL did not label sensory neurons at all. SJA is reported to prefer $\beta$-linked galNac (Poretz et al., 1974), which most likely accounts for its failure to label sensory neurons expressing $\alpha$-linked galNac. Perhaps LBL also selectively recognizes $\beta$-linked galNac. Alternatively, LBL might be prevented from binding to galNac on sensory neurons by steric or local environmental hindrances. The more subtle differences seen in binding between the lectins in Group D, Table 1, suggest that the various lectins recognize slightly different aspects of the local environment of the monosaccharide, which affects their ability to bind. This is not surprising since lectins with "identical" monosaccharide specificity have varying abilities to agglutinate human red blood cells (Debray et al., 1981).

As previously reported (Hamburger and Levi-Montalcini, 1949), the neurons in DRGs of week-old chicks did not fall into 2 distinct size classes, although the size distribution was skewed toward the smaller-diameter cells. Darkly stained, DBA-positive cells clearly fell into this category. There was some variability in the absolute size of DBA-labeled neurons among the 3 chicks studied. One explanation for this is that chick $L 40$ in Figure 3 appeared to be relatively less mature than the other 2 chicks, as judged by the absence of DBA staining in dorsal horn lamina 3. This could account for the overall smaller size of its DRG neurons (cf. Lawson, 1979). Nevertheless, considering the different techniques used to measure neuron sizes and the subjectivity involved in scoring the intensity of DBA staining in neurons, the correspondence among embryos is striking.

The organization of the chick dorsal horn differs from that of mammals, with laminae 1 and 2 lying lateral rather than dorsal to lamina 3 (Martin, 1979). The laminar arrangements of central projections of sensory neurons have not been mapped in detail in the chick to detcrminc whether the terminals of different modalities are segregated in laminae corresponding to those in mammals. If this is the case, then the size of the labeled neurons and the location of their projections in the dorsal horn suggest that the subset of neurons that express terminal galNac residues could be nociceptive, thermoreceptive, or high-threshold mechanoreceptive neurons (Sugiura et al., 1986), but are unlikely to be low-threshold cutaneous or muscle afferents (Brown, 1981). It remains to be determined whether the carbohydrate(s) recognized by DBA in the chick defines a single functional class of sensory neurons or is characteristic of all classes of neurons that project to superficial dorsal horn laminae.

An analogous population of neurons appears to be identified by unique glycoconjugates in a number of species. For example, in the rat most small-diameter DRG neurons and their terminals in laminae I and II can be stained with lectins specific for $\alpha$-galactose residues (Streit et al., 1985; Nakagawa et al., 1986), while in the rabbit (Mori, 1986) and humans (Mori, 1986; Nakagawa et al., 1986) a seemingly similar population of neurons expresses terminal fucose residues. The glycoconjugates are not the same in these 2 species, however, since the lectin-binding moiety appears to be a glycolipid in the rabbit (Mori, 1986) and a glycoprotein in humans (Nakagawa et al., 1986). Nociceptive neurons in the cat, as in the chick, express terminal galNac residues (Plenderleith et al., 1988). Again, the diagnostic glycoconjugates differ between the 2 species, however. GalNac residues can be labeled in paraffin-embedded sections in the cat, suggesting that the monosaccharide is associated with a glycoprotein. By contrast, we found that galNac labeling in the chick is completely removed by paraffin-embedding techniques, as if galNac were associated with a glycolipid. The reason for this heterogeneity in the glycoconjugate signature among seemingly homologous populations of neurons in different species is enigmatic. It will be of interest to see whether the different carbohydrates serve a similar function in all labeled neurons.

The sensory neurons identified by galNac-binding lectins correspond in size and location to the population of DRG neurons that express SP, CGRP (Ncw and Mudge, 1986; Du et al., 1987), myelin-associated glycoprotein (MAG) (Omlin et al., 1985), and the lactoseries glycoconjugates AC4 antigen and SSEA-1 (Scott et al., 1987). The extent to which these markers coexist in individual neurons is unknown.

The glycoconjugate described here appears much later in development than SP (New and Mudge, 1986; Du et al., 1987), MAG (Philippe et al., 1986), or lactoseries glycoconjugates (Scott et al., 1987). Thus, its expression is likely to be regulated differently, and it undoubtedly serves a different function from the earlier-appearing glycoconjugates and peptides. The developmental regulation and function of the DBA-binding glycoconjugate on chick sensory neurons, however, remain to be determined.

\section{References}

Altman, J., and S. A. Bayer (1984) The development of the rat spinal cord. In Advances in Anatomy, Embryology, and Cell Biology, vol. 85 , F. Beck, W. Hild, J. van Limborgh, R. Ortman, J. E. Pauly, and T. H. Schiebler, eds., Springer-Verlag, Berlin.

Brown, A. G. (1981) Organization in the Spinal Cord, Springer-Verlag, New York.

Davis, B. M., E. Frank, F. A. Johnson, and S. A. Scott (1989) The development of central projections of lumbosacral sensory neurons in the chick. J Comp. Neurol. 279: 556-566.

Debray, H., D. Decout, G. Strecker, G. Spik, and J. Montreuil (1981) Specificity of twelve lectins towards oligosaccharides and glycopeptides related to $N$-glycosylproteins. Eur. J. Biochem. 117: 41-55.

Dodd, J., and T. M. Jessell (1985) Lactoseries carbohydrates specify subsets of dorsal root ganglion neurons projecting to the superficial dorsal horn of rat spinal cord. J. Neurosci. 5: 3278-3294.

Dodd, J., and T. M. Jessell (1986) Cell surface glycoconjugates and carbohydrate-binding protcins: Possible recognition signals in sensory neurone development. J. Exp. Biol. 124: 225-238.

Dodd, J., D. Solter, and T. M. Jessell (1984) Monoclonal antibodies against carbohydrate differentiation antigens identify subsets of primary sensory neurons. Nature 311: 469-472.

Du, F., Y. Charnay, and P. Dubois (1987) Development and distribution of substance $P$ in the spinal cord and ganglia of embryonic and newly hatched chick: An immunofluorescence study. J. Comp. Neurol. 263: 436-454.

Edelman, G. M. (1984) Modulation of cell adhesion during induction, histogenesis, and perinatal development of the nervous system. Annu. Rev. Neurosci. 7: 339-377.

Fischer, J., and B. Csillik (1985) Lectin binding: A genuine marker for transganglionic regulation of human primary sensory neurons. Neurosci. Lett. 54: 263-267.

Fraser, S. E., M. S. Carhart, B. A. Murray, C.-M. Chuong, and G. M. Edelman (1988) Alterations in the Xenopus retinotectal projection by antibodies to Xenopus N-CAM. Dev. Biol. 129: 217-230.

Galbraith, W., and I. J. Goldstein (1970) Phytohemagglutinins: A new class of metalloproteins. Isolation, purification, and some properties of the lectin from Phaseolus lunatus. FEBS Lett. 9: 197-201.

Grunwald, G. B., P. Fredman, J. L. Magnani, D. Trisler, V. Ginsburg, and $M$. Nirenberg (1985) Monoclonal antibody 18B8 detects gangliosides associated with neuronal differentiation and synapse formation. Proc. Natl. Acad. Sci. USA 82: 4008-4012.

Hamburger, V., and H. L. Hamilton (1951) A series of normal stages in the development of the chick embryo. J. Morphol. 88: 49-92.

Hamburger, V., and R. Levi-Montalcini (1949) Proliferation, differentiation and degeneration in the spinal ganglia of the chick embryo under normal and experimental conditions. J. Exp. Zool. 111: 457502.

Hammarstrom, S., L. A. Murphy, I. J. Goldstein, and M. E. Etzler 
(1977) Carbohydrate binding specificity of four $N$-acetyl-D-galactosamine-"specific" lectins: Helix pomatia A hemagglutinin, soy bean agglutinin, lima bean lectin, and Dolichos biflorus lectin. Biochemistry 16: $2750-2755$.

Harper, A. A., and S. N. Lawson (1985) Flectrical properties of rat dorsal root ganglion neurones with different peripheral nerve conduction velocities. J. Physiol. 359: 47-63.

Hökfelt, T., R. Elde, O. Johansson, R. Luft, G. Nilsson, and A. Arimura (1976) Immunohistochemical evidence for separate populations of somatostatin-containing and substance $\mathrm{P}$-containing primary afferent neurons in the rat. Neuroscience $1:$ 131-136.

Jessell, T. M., and J. Dodd (1985) Structure and expression of differentiation antigens on functional subclasses of primary sensory neurons. Philos. Trans. R. Soc. Lond. (Biol.) 308: 271-281.

Kannagi, R., S. B. Levery, F. Ishigami, S. Hakomori, L. H. Shevinsky, B. B. Knowles, and D. Solter (1983) New globoseries glycosphingolipids in human teratocarcinoma reactive with the monoclonal antibody directed to a developmentally regulated antigen, stage-specific embryonic antigen 3. J. Biol. Chem. 258: 8934-8942.

Landmesser, L., L. Dahm, K. Schultz, and U. Rutishauser (1988) Distinct roles for adhesion molecules during innervation of chick muscle. Dev. Biol. 130: 645-670.

Lawson, S. N. (1979) The postnatal development of large light and small dark neurones in mouse dorsal root ganglia: A statistical analysis of cell numbers and size. J. Neurocytol. 8: 275-294.

Lawson, S. N., E. I. Harper, A. A. Harper, J. A. Garson, H. B. Coakham, and B. J. Randle (1985) Monoclonal antibody 2C5: A marker of a subpopulation of small neurones in rat dorsal root ganglia. Neuroscience 16: 365-374.

Leah, J. D., A. A. Cameron, and P. J. Snow (1985) Neuropeptides in physiologicaly identified mammalian sensory neurones. Neurosci. Lett. 56: $257-263$.

I ee, M. T., M. J. Koebbe, and M. J. O'Donovan (1988) The development of sensorimotor synaptic connections in the lumbosacral cord of the chick embryo. J. Neurosci. 8: 2530-2543.

Levine, J. M., L. Beasley, and W. B Stallcup (1984) The D1.1 antigen: A cell surface marker for germinal cells of the central nervous system. J. Neurosci. 4: 820-831.

Lieberman, A. R. (1976) Sensory ganglia. In The Peripheral Nerve, D. N. Landon, ed., pp. 188-278, Wiley, New York.

Martin, A. H. (1979) A cytoarchitectonic scheme for the spinal cord of the domestic fowl, Gallus gallus domesticus: Lumbar region. Acta Morphol. Neerl. Scand. 17: 105-117.

Marusich, M. F., K. Pourmehr, and J. A. Weston (1986) A monoclonal antibody (SN1) identifies a subpopulation of avian sensory neurons whose distribution is correlated with axial level. Dev. Biol. 118:494504

Mori, K. (1986) Lectin Ulex europaeus agglutinin I specifically labels a subset of primary afferent fibers which project selectively to the superficial dorsal horn of the spinal cord. Brain Res. 365: $404-408$.

Nakagawa, F., B. A. Schulte, and S. S. Spicer (1986) Lectin cytochemical evaluation of somatosensory neurons and their peripheral and central processes in rat and man. Cell Tissue Res. 245: 579-589.

New, H. V., and A. W. Mudge (1986) Distribution and ontogeny of SP, CGRP, SOM, and VIP in chick sensory and sympathetic ganglia. Dev. Biol. 116: 337-346.

Omlin, F. X., J.-M. Matthieu, E. Philippe, J.-M. Roch, and B. Droz (1985) Expression of myelin-associated glycoprotein by small neurons of the dorsal root ganglion in the chicken. Science 227: 13591360.

Patel, N., and S. A. Scott (1988) Lectin binding to glycoconjugates in chick spinal cord and dorsal root ganglia. Neurosci. Abstr. 14: 393.

Peinado, A., E. R. Macagno, and B. Zipser (1987) A group of related surface glycoproteins distinguish sets and subsets of sensory afferents in the leech nervous system. Brain Res. 410: 335-339.

Philippe, E., F. X. Omlin, and B. Droz (1986) Myelin-associated glycoprotein immunoreactive material: An early neuronal marker of dorsal root ganglion cells during chick development. Dev. Brain Res. 27: 275-277.

Plenderleith, M. B., A. A. Cameron, B. Key, and P. J. Snow (1988) Soybean agglutinin binds to a subpopulation of primary sensory neurones in the cat. Neurosci. Lett. 86:257-262.

Poretz, R. D., H. Riss, J. W. Timberlake, and S. Chen (1974) Purification and properties of the hemagglutinin from Sophora japonica seeds. Biochemistry 13: 250-256.

Price, J. (1985) An immunohistochemical and quantitative examination of dorsal root ganglion neuronal subpopulations. J. Neurosci. 5: 2051-2059.

Rambourg, A., Y. Clermont, and A. Beaudet (1983) Ultrastructural features of six types of neurons in rat dorsal root ganglia. J. Neurocytol. 12: 47-66.

Rose, R. D., and D. Rohrlich (1987) Counting sectioned cells via mathematical reconstruction. J. Comp. Neurol. 263: 365-386.

Rose, R. D., H. R. Koerber, M. J. Sedivec, and L. M. Mendell (1986) Somal action potential duration differs in identified primary afferents. Ncurosci. Lett. 63: 259-264.

Schwarting, G. A., F. B. Jungalwala, D. K. H. Chou, A. M. Boyer, and M. Yamamoto (1987) Sulfated glucuronic acid-containing glycoconjugates are temporally and spatially regulated antigens in the developing nervous system. Dev. Biol. 120: 65-76.

Scott, L. J. C., F. Bacou, and J. R. Sanes (1988) A synapse-specific carbohydrate at the neuromuscular junction: Association with both acetylcholinesterase and a glycolipid. J. Neurosci. 8: 932-944.

Scott, S. A. (1987) The development of skin sensory innervation patterns. Trends Neurosci. 10: 468-473.

Scott, S. A., J. Dodd, and T. M. Jessell (1987) Ontogeny of carbohydrate differentiation antigens in chick dorsal root ganglia and spinal cord. Neurosci. Abstr. 13: 78.

Shevinsky, L. H., B. B. Knowles, I. Damjanov, and D. Solter (1982) Monoclonal antibody to murine embryos defines a stage-specific embryonic antigen expressed on mouse embryos and human teratocarcinoma cells. Cell 30: 697-705.

Smith, C. L. (1983) The development and postnatal organization of primary afferent projections to the rat thoracic spinal cord. J. Comp. Neurol. 220: 29-43.

Smith, C. L., and E. Frank (1988) Specific sensory projections to the spinal cord during development in bullfrogs. J. Comp. Neurol. 269: 96-108.

Sommer, E. W., J. Kazimierczak, and B. Droz (1985) Neuronal subpopulations in the dorsal root ganglion of the mouse as characterized by combination of ultrastructural and cytochemical features. Brain Res. 346: 310-326.

Sternberger, L. A. (1986) Immunocytochemistry, 3rd ed., Wiley, New York

Streit, W. J., B. A. Schulte, J. D. Balentine, and S. S. Spicer (1985) Histochemical localization of galactose-containing glycoconjugates in sensory neurons and their processes in the central and peripheral nervous system of the rat. J. Histochem. Cytochem. 33: 1042-1052.

Streit, W. J., B. A. Schulte, J. D. Balentine, and S. S. Spicer (1986) Evidence for glycoconjugate in nociceptive primary sensory neurons and its origin from the Golgi complex. Brain Res. 377: 1-17.

Sugiura, Y., C. L. Lee, and E. R. Perl (1986) Central projections of identified, unmyelinated (C) afferent fibers innervating mammalian skin. Science 234: 358-361.

Yamamoto, M., A. M. Boyer, and G. A. Schwarting (1985) Fucosecontaining glycolipids are stage- and region-specific antigens in developing embryonic brain of rodents. Proc. Natl. Acad. Sci. USA 82: 3045-3049. 